\title{
A Higher-order Explicit Marching-on-in-time for Analysis of Transient Acoustic Scattering from Rigid Objects
}

\author{
Rui Chen and Hakan Bagci \\ Division of Computer, Electrical, and Mathematical Science and Engineering (CEMSE) \\ King Abdullah University of Science and Technology (KAUST), Thuwal, 23955-6900, Saudi Arabia \\ e-mails: $\{$ rui.chen, hakan.bagci\}@kaust.edu.sa
}

\begin{abstract}
An explicit marching-on-in-time (MOT) scheme for solving the time domain Kirchhoff (surface) integral equation (TDKIE) to analyze acoustic scattering from a rigid scatterer is presented. The unknown velocity potential induced on the scatterer surface is approximated using nodal interpolation functions in space and shifted Lagrange polynomials in time. Inserting this expansion into the TDKIE and testing the resulting equation at the interpolation points (i.e., Nyström method in space) yield a system of ordinary differential equations (ODEs). This ODE system is integrated in time using a $\mathrm{PE}(\mathrm{CE})^{m}$-type linear multistep scheme to yield unknown expansion coefficients. Numerical results demonstrate that the explicit MOT scheme uses the same time step as its implicit counterpart without sacrificing from accuracy or stability and is faster under low frequency excitation (i.e., for large time step).
\end{abstract}

\section{INTRODUCTION}

Transient acoustic scattering from rigid scatterers can be analyzed using either differential equation solvers or time domain (surface) Kirchhoff integral equation (TDKIE) [1] solvers. The surface integral equation solvers have several advantages over the differential equation solvers: They discretize only the surface of the scatterer, are free from numerical dispersion, and do not need absorbing boundary conditions to truncate the computation domain. The TDKIE is often solved using a marching-on-in-time (MOT) scheme [1], which can be either implicit or explicit depending on the types of spatial and temporal basis functions used for expanding the unknown surface velocity potential, type of the testing scheme, and the time step size. Unlike their implicit counterparts, traditional explicit MOT schemes do not call for a matrix inversion at every time step of the time marching. However, they require smaller time steps to maintain the stability of the solution (i.e., their time step size is subject to Courant-FriedrichsLewy (CFL) constraint) resulting in longer simulation times. Recently, a quasi-explicit MOT scheme [2] has been developed to overcome this drawback. This scheme uses RaoWilton-Glisson (RWG) functions [3] to spatially discretize the time domain magnetic field integral equation (TDMFIE) (in unknown electric current) and integrates the resulting system of ODEs for the coefficients of the current expansion using a $\mathrm{PE}(\mathrm{CE})^{m}$-type linear multistep scheme. The resulting marching scheme still requires solving a matrix system at every time step, however unlike its implicit counterpart, the matrix is always sparse and well-conditioned regardless of the time step size. Additionally, both schemes use the same time step size, which consequently makes the explicit solver faster.

In this work, a fully explicit MOT scheme is developed for solving the TDKIE for analyzing transient acoustic scattering from rigid objects. The surface velocity potential is expanded using higher-order polynomial interpolation functions [4] in space instead of overlapping basis functions (such as RWG functions). Inserting this expansion into the TDKIE and point testing the resulting equation at the interpolation points (i.e., Nyström method in space) yields system with a diagonal matrix. This completely eliminates the need for a matrix inversion. Numerical results demonstrate that the proposed explicit solver can use the same time step as its implicit counterpart without sacrificing from accuracy or stability and is faster under low frequency excitation (i.e., for large time step).

\section{FORMULATION}

Let $S$ denote the surface of an acoustically rigid scatterer residing in an unbounded homogeneous medium. A band-limited acoustic excitation with velocity potential $\varphi^{\mathrm{i}}(\mathbf{r}, t)$ impinges on $S$. Using Kirchhoff-Helmholtz theorem and enforcing the boundary condition for total pressure field on $S$, one obtains the TDKIE in unknown total velocity potential $\varphi(\mathbf{r}, t)$ as [1]:

$$
\begin{aligned}
\frac{1}{2} \partial_{t} \varphi(\mathbf{r}, t) & -\partial_{t} \int_{S} \varphi\left(\mathbf{r}^{\prime}, t\right) * \hat{\mathbf{n}}\left(\mathbf{r}^{\prime}\right) \cdot \nabla^{\prime} \frac{\delta(t-R / c)}{4 \pi R} d s^{\prime} \\
& =\partial_{t} \varphi^{\mathrm{i}}(\mathbf{r}, t) .
\end{aligned}
$$

Here, $R=\left|\mathbf{r}-\mathbf{r}^{\prime}\right|$ is the distance between the observation point $\mathbf{r}$ and the source point $\mathbf{r}^{\prime}, \hat{\mathbf{n}}\left(\mathbf{r}^{\prime}\right)$ is the outward pointing unit normal to $S$ at $\mathbf{r}^{\prime}$, and $c$ is the speed of sound in the background medium. Note that the integral in (1) should be evaluated in the principal value sense. To numerically solve (1), $S$ is discretized into $N_{\mathrm{p}}$ curvilinear triangular patches and $\varphi(\mathbf{r}, t)$ is expanded using interpolation functions $L_{i p}(\mathbf{r})$ as [4]:

$$
\varphi(\mathbf{r}, t)=\sum_{p=1}^{N_{\mathrm{p}}} \sum_{i=1}^{N_{\mathrm{i}}}\{\mathbf{I}(t)\}_{i p} \vartheta(\mathbf{r}) L_{i p}(\mathbf{r}) .
$$

Here, $N_{\mathrm{i}}$ is the number of interpolation points on each patch, $\vartheta(\mathbf{r})$ is the inverse of the Jacobian, $\{\mathbf{I}(t)\}_{i p}$ is the unknown time-dependent coefficient of $L_{i p}(\mathbf{r})$, and $L_{i p}(\mathbf{r})$ is 
the Lagrange interpolator defined at $\mathbf{r}_{i p}\left(i^{\text {th }}\right.$ interpolation point on $p^{\text {th }}$ patch). Substituting (2) in (1) and point testing the resulting equation at $\mathbf{r}_{j q}, j=1, \ldots, N_{\mathrm{i}}, q=1, \ldots, N_{\mathrm{p}}$, yield a time-dependent linear matrix system. Sampling this system at times $t=k \Delta t, k=1, \ldots, N_{\mathrm{t}}$, and using Lagrange polynomial functions $T(t)$ [1] to facilitate the evaluation of the retarded time integrals result in a fully discretized system:

$$
\mathbf{G i}_{k}=\mathbf{V}_{k}^{\mathrm{i}}+\sum_{l=1}^{k} \mathbf{Z}_{k-l} \mathbf{I}_{l}, \quad k=1, \ldots, N_{\mathrm{t}} .
$$

Here, $\{\mathbf{G}\}_{j q, i p}=\frac{1}{2} \vartheta\left(\mathbf{r}_{j q}\right) \delta_{i j} \delta_{p q},\left\{\dot{\mathbf{I}}_{k}\right\}_{i p}=\left\{\left.\partial_{t} \mathbf{I}(t)\right|_{t=k \Delta t}\right\}_{i p}$, $\left\{\mathbf{V}_{k}^{\mathrm{i}}\right\}_{j q}=\partial_{t} \varphi^{\mathrm{i}}\left(\mathbf{r}_{j q}, k \Delta t\right),\left\{\mathbf{I}_{l}\right\}_{i p}=\{\mathbf{I}(l \Delta t)\}_{i p}$, and

$$
\begin{aligned}
& \left\{\mathbf{Z}_{k-l}\right\}_{j q, i p}= \\
& \left.\quad \int_{S_{p}} \vartheta\left(\mathbf{r}^{\prime}\right) L_{i p}\left(\mathbf{r}^{\prime}\right) \hat{\mathbf{n}}^{\prime}\left(\mathbf{r}^{\prime}\right) \cdot \nabla^{\prime} \frac{\partial_{t} T(t)}{4 \pi R}\right|_{t=(k-l) \Delta t-R / c} d s^{\prime}
\end{aligned}
$$

where $R=\left|\mathbf{r}_{j q}-\mathbf{r}^{\prime}\right|$ and $\delta_{i j}=1$ for $i=j$, and $\delta_{i j}=0$ for $i \neq j$. The ODE system (3) is integrated in time using a $\mathrm{PE}(\mathrm{CE})^{m}$-type multistep method enhanced with a successiveover-relaxation (SOR) scheme [5] to obtain $\mathbf{I}_{k}, k=1, \ldots, N_{\mathrm{t}}$. The resulting explicit MOT scheme requires the solution of (3) at every time step. This is a trivial task since $\mathbf{G}$ is diagonal with entries equal to $\vartheta\left(\mathbf{r}_{j q}\right) / 2$.

\section{NUMERICAL RESUlTS}

In this section, the proposed explicit MOT scheme and its implicit counterpart are used to analyze acoustic scattering from a rigid unit sphere residing in water with $c=1500 \mathrm{~m} / \mathrm{s}$. In all the simulations, $\varphi^{\mathrm{i}}(\mathbf{r}, t)=G(t-z / c)$, where $G(t)=$ $\cos \left[2 \pi f_{0}\left(t-t_{\mathrm{p}}\right)\right] e^{-\left(t-t_{\mathrm{p}}\right)^{2} / 2 w^{2}}$ is the modulated Gaussian pulse, $t_{\mathrm{p}}=6 \mathrm{w}$ and $w$ are the pulse delay and width, and $f_{0}$ is the modulation frequency. The Nyström method uses second-order interpolation functions $\left(N_{\mathrm{i}}=6\right)$ and $T(t)$ is a third-order Lagrange polynomial function. The matrix system of the implicit scheme is iteratively solved at every time step using the transpose-free quasi-minimal residual method [6]. Both schemes use the same accuracy tolerance of $10^{-13}$.

For the first example, $N_{\mathrm{p}}=2560$ (resulting in $N_{\mathrm{s}}=15360$ unknowns), $f_{0}=700 \mathrm{~Hz}, w=2.183 \mathrm{~ms}, \Delta t=0.0293 \mathrm{~ms}$ and $N_{\mathrm{t}}=1400$. The predictor and corrector steps use AdamsBashforth and backward difference methods, respectively [7], and the SOR scheme is not utilized. Fig. 1 compares $\left|\{\mathbf{I}(t)\}_{i p}\right|$ , $p=51, i=5$, computed by the explicit and implicit schemes at $\mathbf{r}_{i p}=(0.0,0.366,-0.931) \mathrm{m}$. Results agree well. Fig. 2 compares the scattering cross section (SCS) obtained from the Mie series and the solution computed by the explicit and implicit solvers at $f=700 \mathrm{~Hz}$ for $\theta=\left[0^{\circ}, 180^{\circ}\right]$ and $\phi=0^{\circ}$. Let $\sigma^{\mathrm{Mie}}, \sigma^{\exp }$, and $\sigma^{\mathrm{imp}}$ represent these SCS values. The figure shows that both solvers provide accurate results. Note that both solvers require the same execution time.

For the second example, $N_{\mathrm{p}}=1126, N_{\mathrm{s}}=6756, f_{0}=$ $45 \mathrm{~Hz}, w=34 \mathrm{~ms}, \Delta t=1.37 \mathrm{~ms}$, and $N_{\mathrm{t}}=400$. The predictor and corrector coefficients are obtained using the method described in [8], and the SOR coefficient is 0.6. The relative $L_{2}$ norm errors in $\sigma^{\exp }$ and $\sigma^{\mathrm{imp}}$ (with respect to $\sigma^{\mathrm{Mie}}$ ) are 0.0044 and 0.0104 , respectively. Note that this is

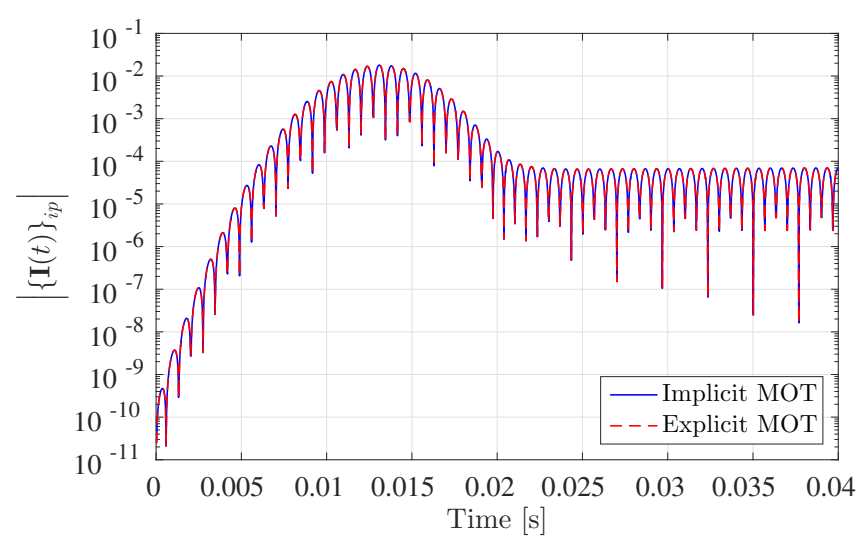

Fig. 1. $\left|\{\mathbf{I}(t)\}_{i p}\right|, p=51, i=5$, computed by the implicit and explicit MOT solvers at $\mathbf{r}_{i p}=(0.0,0.366,-0.931) \mathrm{m}$.

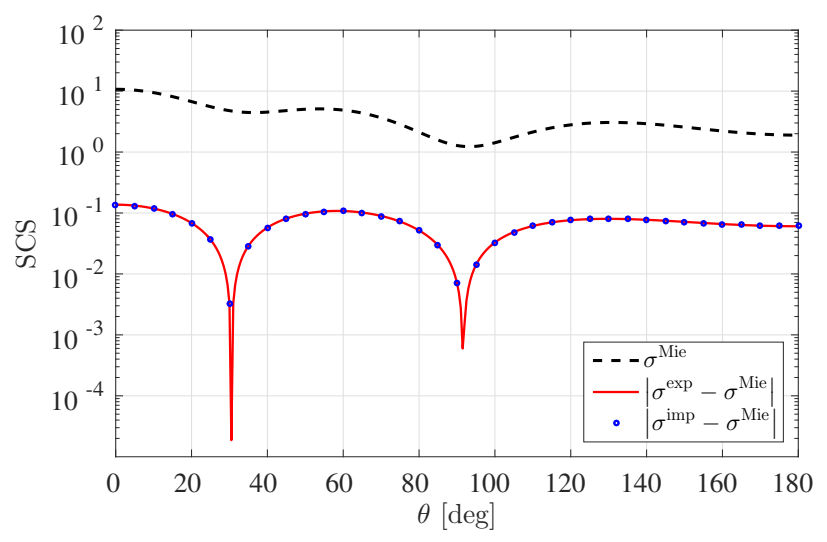

Fig. 2. SCS obtained at $f=700 \mathrm{~Hz}$ for $\theta=\left[0^{\circ}, 180^{\circ}\right]$ and $\phi=0^{\circ}$ from the solutions computed by the Mie series and the implicit and explicit MOT solvers.

a low frequency problem (i.e., large $\Delta t$ ), where the implicit scheme solves a full matrix system at every time step. The proposed explicit scheme is almost two times faster.

\section{REFERENCES}

[1] A. A. Ergin, B. Shanker, and E. Michielssen, "Analysis of transient wave scattering from rigid bodies using a Burton-Miller approach," J. Acoust. Soc. Am., vol. 106, pp. 2396-2404, Nov. 1999.

[2] H. A. Ulku, H. Bagci, and E. Michielssen, "Marching on-in-time solution of the time domain magnetic field integral equation using a predictorcorrector scheme," IEEE Trans. Antennas Propag., vol. 61, pp. 41204131, Aug. 2013.

[3] S. M. Rao, D. R. Wilton, and A. W. Glisson, "Electromagnetic scattering by surfaces of arbitrary shape", IEEE Trans. Antennas Propag., vol. 30, pp. 408-418, May 1982.

[4] G. Kang, J. Song, W. C. Chew, K. C. Donepudi, and J.-M. Jin, "A novel grid-robust higher order vector basis function for the method of moments," IEEE Trans. Antennas Propag., vol. 49, pp. 908-915, 2001.

[5] S. B. Sayed, H. A. Ulku, and H. Bagci, "Stable quasi-explicit MOT solver for the time domain volume electric field integral equation," in Proc. Appl. Comput. Electromagn. Symp., pp. 416-420, Mar. 2014.

[6] R. W. Freund, "A transpose-free quasi-minimal residual algorithm for non-Hermitian linear systems," SIAM J. Sci. Comput., vol. 14, pp. 470482, Mar. 1993.

[7] E. Hairer, S. P. Norsett, and G. Warner, Solving Ordinary Differential Equations I: Nonstiff Problems. Springer, New York, 2010.

[8] A. Glaser and V. Rokhlin, "A new class of highly accurate solvers for ordinary differential equations," J. Sci. Comput., vol. 38, pp. 368-399, Mar. 2009. 